\title{
A NEW APPROACH TO STOCHASTIC INTEGRATION WITH RESPECT TO FRACTIONAL BROWNIAN MOTION FOR NO ADAPTED PROCESSES
}

\author{
BACHIR CHERIF KHALIDA ${ }^{1, a}$ AND KANDOUCI ABDELDJEBBAR ${ }^{2, b}$
}

\footnotetext{
${ }^{1}$ Laboratory of Stochastic Models, Statistic and Applications, University of Saida Dr Moulay Tahar. Algeria.

${ }^{a}$ E-mail: Khalida.bachircherif@univ-saida.dz

${ }^{2}$ Laboratory of Stochastic Models, Statistic and Applications, University of Saida Dr Moulay Tahar. Algeria.

${ }^{b}$ E-mail: abdeldjebbar.kandouci@univ-saida.dz or kandouci1974@yahoo.fr

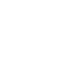

\section{Abstract}

In this paper, we propose a new approach to stochastic integration of the class of instantly independent stochastic processes with respect to fractional Brownian motion on a finite interval. The appraisal point is to discover the counterpart of the Itô theory. More precisely, we show some result on stochastic integration with respect to no adapted processes by generalizing the results obtained by Ayed and Kuo [5] in the Brownian framework.

\section{Introduction}

Fractional Brownian motion was introduced by Kolmogorov [14] while studying spiral curves in Hilbert space. Later, its properties were given by Mandelbrot and Van Ness [17]. In fact, in [17], authors considered the fractional Brownian motion as a centered and continuous Gaussian process, denoted by $B^{H}=\left\{B_{t}^{H}, t \geq 0\right\}, H \in(0,1)$ with covariance

$$
\mathbb{E}\left(B_{t}^{H}, B_{s}^{H}\right)=\frac{V_{H}}{2}\left(t^{2 H}+s^{2 H}-(t-s)^{2 H}\right),
$$

Received June 30, 2021.

AMS Subject Classification: 60G15.

Key words and phrases: Fractional Brownian motion, Lévy-Hida representation, stochastic integration, Gaussian measure, Instantly independent processes. 
and $V_{H}$ is normalizing constant given by

$$
V_{H}=\frac{\Gamma(2-2 H) \cos (\pi H)}{\pi H(1-2 H)} .
$$

This process is starting from 0 with stationary increments, $\mathbb{E}\left(B_{t}^{H}-B_{s}^{H}\right)=$ $V_{H}|t-s|^{2 H}$, which is self-similar, and, $B_{a t}^{H}$ has the same distribution as $a^{H} B_{t}^{H}$. The parameter $H$ determines the sign of the covariance of past increments and the future. This latter is positive when $H>\frac{1}{2}$ and negative when $H<\frac{1}{2}$. Moreover, it exhibits a long-range dependence in the sense that the covariance between increments at a distance $u$ decreases to zero as $u^{2 H-2}$.

The self-similarity and long-range dependence properties make the fractional Brownian motion a suitable driving noise in different applications like economics, finance, and telecommunications especially in internet traffic to hydrology problems via linguistic.

Lin [19] also Dai and Heyde [21] gave a stochastic integral $\int_{0}^{t} \phi_{s} d B_{s}^{H}$ as limit of Riemann sums. The propriety $\mathbb{E}\left(\int_{0}^{t} \Phi_{s} d B_{s}^{H}\right)=0$ is not satisfying by this integral, Duncan et al. [20] introduced a new stochastic integral with zero means which is the limit of Riemann sums defined by means of the Wick product [1]. Alo et al.(2000) [1] constructed a stochastic integral with respect to the fBm with Hurst parameter lesser than 0, and in 2010 Ayed and Kuo [5] explained this idea for an extension of the Itô integral.

Our aim in this paper is to introduce a new approach of stochastic integration for processes not necessarily adapted with respect to fractional Brownian motion. Particulary, we are interested in the case when the index $H$ is greater then $\frac{1}{2}$.

This paper is organized as follow : In section 1, we recall some preliminaries on Malliavin calculus and on the fractional calculus. In section 2, we construct suitable spaces of integrands in order to have a well-defined integral using integral representation. In section 3, We will introduce a new outcome on stochastic integration w.r.t fractional Brownian motion (fbm) for non adapted process by using an idea of Lebovits [16], and we give a new result on stochastic integration w.r.t. fbm for no adapted processes that are 
written as the product of two processes, one is adapted, and the second is instantly independent.

\section{Preliminaries}

Let $\mathcal{F}_{t}, 0 \leq a \leq t \leq b \leq T$ a filtration satisfying the following conditions

- (a) $B_{t}$ is $\{\mathcal{F}\}$-adapted i.e. $B_{t}$ is $\mathcal{F}_{t}$ measurable for each $t \in[a, b]$.

- (b) $\left(B_{t}-B_{s}\right)$ and $\mathcal{F}_{s}$ are independent for any $s \leq t \in[a, b]$.

Let $\tilde{\mathcal{H}}$ be a some class of integrands and complete, and let $\mathcal{E} \in \tilde{\mathcal{H}}$ be the class of step functions, and $\mathcal{J}^{H}(f)$ be an integral of $f \in \mathcal{E}$ w.t.r. fractional Brownian motion $B_{t}^{H}$, under these assumptions:

- $\tilde{\mathcal{H}}$ is an inner product space with an inner product $<f, g>_{\tilde{\mathcal{H}}}, f, g \in \tilde{\mathcal{H}}$.

- for $f, g \in \mathcal{E},\left\langle f, g>_{\tilde{\mathcal{H}}}=\mathbb{E}\left[\mathcal{J}^{H}(f) . \mathcal{J}(g)\right]\right.$.

- The set $\mathcal{E}$ is dense in $\tilde{\mathcal{H}}$.

In this section, we give a short summary of fractional calculus for processes driven by fractional Brownian motion.

Fractional calculus is a branch of mathematical analysis that unifies the integration operator and differentiation operator of classical calculus as one operator, the differintegral, which is a single operator depending on a real valued parameter $\alpha$, where the positive values of $\alpha$ correspond to differentiation and negative values of $\alpha$ correspond to integration. Fractional calculus is an extension, or generalization, of the well known classical calculus[2]. It was presented by B.W.Leibniz in (1695), as be mentioned by J.Wallis the possible approach to fractional-order differentiation in that sense, for non-integer values of $n$ the definition is given as

$$
\frac{d^{n} e^{x m}}{d x^{n}}=m^{n} e^{m x}
$$

Authors suggested to use this relationship also for negative or non-integer (rational) values of $n$, and they generalized the notion of differentiation for arbitrary functions. 
According to Riemann-Liouville, the notion of fractional integral of order $\alpha,(\alpha>0)$ for a function $f(t)$, is a natural consequence of the well known formula (Cauchy-Dirichlet ) that reduces the calculation of the $n$-fold primitive of a function $f(t)$ to a single integral of convolution type

$$
\mathcal{J}_{a+}^{n} f(t)=\frac{1}{(n-1) !} \int_{a}^{t}(t-\tau)^{n-1} f(\tau) d \tau, \quad n \in \mathbb{N}
$$

disappear at $t=a$ with its derivative $1,2,3, \ldots, n-1$. Impose $\mathcal{J}_{a+}^{n} f(t)$ and $f(t)$ to be causal function, here, vanishing for $t<0$. Expand to any positive real value by using the Gamma function $(n-1) !=\Gamma(n)$.

\subsection{Fractional Integral of order $\alpha>0$ (Right-Sided)}

We have

$$
\mathcal{J}_{a+}^{\alpha} f(t)=\frac{1}{\Gamma(n)} \int_{a}^{t}(t-\tau)^{\alpha-1} f(\tau) d \tau, \quad \alpha \in \mathbb{R} .
$$

We define $\mathcal{J}_{a+}^{0}=I, \mathcal{J}_{a+}^{0} f(t)=f(t)$.

\section{Alternatively(left-sided integral)}

$$
\mathcal{J}_{b-}^{\alpha} f(t)=\frac{1}{\Gamma(n)} \int_{t}^{b}(t-\tau)^{\alpha-1} f(\tau) d \tau, \quad \alpha \in \mathbb{R} .
$$

For $(a=0, b=+\infty)$, we have the Riemann derivative, and for $(a=-\infty, b=$ $+\infty)$, we have the Liouville derivative. According to [2], we have the following definition

Definition 1. Let $f \in L^{2}[a, b] \subset L^{2}, 0<\alpha<1$ and $t \in[a, b]$. The fractional derivatives of order $\alpha$ on the interval $[a, b]$ are

and

$$
\left(D_{a+}^{\alpha} f\right)(t)=\frac{1}{\Gamma(1-\alpha)} \frac{d}{d u} \int_{a}^{b} f(u)(u-t)_{+}^{-\alpha} d u
$$

$$
\left(D_{b-}^{\alpha} f\right)(t)=\frac{1}{\Gamma(1-\alpha)} \frac{d}{d u} \int_{a}^{b} f(u)(u-t)_{+}^{-\alpha} d u .
$$

Furthermore, this case admits what is known as the Weyl representation of 
the fractional derivatives:

and

$$
\left(D_{a+}^{\alpha} f\right)(t)=\frac{1}{\Gamma(1-\alpha)}\left[\frac{f(t)}{(t-a)^{\alpha}}+\alpha \int_{a}^{t} \frac{f(t)-f(u)}{(t-u)^{\alpha-1}} d u\right]
$$

$$
\left(D_{b-}^{\alpha} f\right)(t)=\frac{1}{\Gamma(1-\alpha)}\left[\frac{f(t)}{(b-t)^{\alpha}}+\alpha \int_{t}^{b} \frac{f(t)-f(u)}{(t-u)^{\alpha-1}} d u\right] .
$$

Notice that $D_{a+}^{\alpha}=I_{a+}^{-\alpha}$ and $D_{b-}^{\alpha}=I_{b-}^{-\alpha}$. Furthermore, the fractional derivatives $D_{a+}^{\alpha}$ and $D_{b-}^{\alpha}$ are called left sided and right sided, respectively.

\subsection{Fractional Brownian motion}

There are another representations of the fractional Brownian motion (fbm) as a Wienner's integral ; Taken $[0, T]$, fbm $\left(B_{t}^{(H)}\right)_{0 \leq t \leq T}$ is defined by a general formula:

$$
B_{t}^{H}=\int_{0}^{t} K_{H}(t, s) d B_{s}, \quad t \in[0, T]
$$

where $\left(B_{t}\right)_{0 \leq t \leq T}$ is one-sided standard Brownian motion satisfying the conditions (a) and (b).

\subsection{Lévy-Hida representation}

Following Decrensfond and $\ddot{U}$ stunel in [13], this Kernel is done as

$$
K_{H}(t, s)=\frac{(t-s)_{+}^{H-\frac{1}{2}}}{\Gamma\left(H+\frac{1}{2}\right)} F\left(\frac{1}{2}-H, H-\frac{1}{2}, H+\frac{1}{2}, 1-\frac{t}{s}\right), 0<s<t<\infty .
$$

and $F$ is Gauss hypergeometric function.

Generally, we have:

$$
R_{H}(t, s)=\int_{0}^{s \wedge t} K_{H}(t, u) K_{H}(s, u) d u
$$

where, $R_{H}(t, s)$ is the covariance function. 
Proposition 1 ([18]). For $H \in\left(\frac{1}{2}, 1\right)$, the Kernel function is given by

$$
K_{H}(t, s)=C_{H} s^{\frac{1}{2}-H} \int_{s}^{t}|u-s|^{H-\frac{3}{2}} u^{H-\frac{1}{2}} d u,
$$

where

$$
C_{H}=\left(\frac{H(2 H-1)}{\beta\left(2-2 H, H-\frac{1}{2}\right)}\right)^{\frac{1}{2}},
$$

with $\beta$ is the beta function:

$$
\beta(a, b)=\int_{0}^{1} t^{a-1}(1-t)^{1-b} d t .
$$

Corollary 1 ([18]). Beside the previous result, we have

$$
\begin{aligned}
R_{H}(t, s)=\left(\varpi_{1}(H)\right)^{2} \int_{0}^{t} & \left\{r^{\frac{1}{2}-H}\left(I_{T-}^{H-\frac{1}{2}} u^{H-\frac{1}{2}} \mathbf{1}_{[0, t)(u)}\right)(r)\right. \\
\times & \left.\left(r^{\frac{1}{2}-H}\left(I_{T-}^{H-\frac{1}{2}} u^{H-\frac{1}{2}} I_{[0, s)(u)}\right)(r)\right)\right\} d r
\end{aligned}
$$

$\varpi_{1}$ is defined by:

$$
\varpi_{1}=\left(\frac{\Gamma\left(H-\frac{1}{2}\right)^{2} H(2 H-1)}{\beta\left(2-2 H, H-\frac{1}{2}\right)}\right)^{\frac{1}{2}} .
$$

Hence, this Kernel can be rewritten as

$$
K_{H}(t, s)=\varpi_{1}(H) s^{\frac{1}{2}-H}\left(I_{T-}^{H-\frac{1}{2}} u^{H-\frac{1}{2}} I_{[0, t)}(u)\right)(s) .
$$

\subsection{Lévy-Hida approach: 18}

By the above condition in last proposition, we have

$$
\frac{\partial K_{H}(t, s)}{\partial t}=C_{H}\left(\frac{t}{s}\right)^{H-\frac{1}{2}}(t-s)^{H-\frac{3}{2}} .
$$

Thus, a linear operator $K_{H}^{*}: \mathcal{E} \longrightarrow \mathbb{L}^{2}[a, b]$ is given by:

$$
\left(K_{H}^{*} \phi\right)(s):=\int_{s}^{T} \phi(t) \frac{\partial K_{H}(t, s)}{\partial t} d t,
$$

where, $\phi \in \mathcal{E}$. 


\subsection{The spaces of stochastic test functions and stochastic distri- butions}

In all this work we denote $\mathcal{S}(\mathbb{R})$ the Schwartz space of rapidly decreasing functions on $\mathbb{R}$ and $\mathcal{S}^{\prime}(\mathbb{R})$ denote its dual space. Let $\mu$ be the standard Gaussian measure on $\mathcal{S}^{\prime}(\mathbb{R})$.

Let $\left(L^{2}\right)=L^{2}\left(\mathcal{S}^{\prime}(\mathbb{R}), \mu\right)$ and let $(\mathcal{S})$ and $(\mathcal{S})^{\star}$ denote the spaces of test functions and generalized functions on $\mathcal{S}^{\prime}(\mathbb{R})$, respectively. Then we have a Geland triple

$$
(\mathcal{S}) \subset\left(L^{2}\right) \subset(\mathcal{S})^{\star}
$$

\section{6. $(\mathcal{S})^{\star}$-process, $(\mathcal{S})^{\star}$-derivative and $(\mathcal{S})^{\star}$-integral}

Denote $\mathcal{B}(\mathbb{R})$ the Borelian $\sigma$-field on $\mathbb{R}$ and $m$ a measure on $\mathcal{B}(\mathbb{R})$ such that $(\mathbb{R}, \mathcal{B}(\mathbb{R}), m)$ is a $\sigma$-finite measure space.

Through this section, $[0, T]$ denote an element of $\mathcal{B}(\mathbb{R})$.

A measurable function $\Phi: I \longrightarrow(\mathcal{S})^{\star}$ is called a stochastic distribution process $\left((\mathcal{S})^{\star}\right.$-process $) . \Phi$ is said differentiable at $t_{0}$ if $\lim _{r \longrightarrow 0} r^{-1}\left(\Phi_{t_{0}+r}-\Phi_{t_{0}}\right)$ exists in $(\mathcal{S})^{\star}$.

One notes $\frac{d \Phi_{t_{0}}}{d t}$ the $(\mathcal{S})^{\star}$-derivative at $t_{0}$ of the stochastic distribution process $\Phi$. If $\Phi$ is differentiable at every $t_{0} \in I$, we said that $\Phi$ is differentiable on $I$. Generally, for every $k \in \mathbb{N}, \Phi$ is $\mathcal{C}^{k}$ in $(\mathcal{S})^{\star}$ if the process $\Phi: I \longrightarrow(\mathcal{S})^{\star}$ is $\mathcal{C}^{k}$.

Definition 2 ([16]). Assume that $\Phi: I \longrightarrow(S)^{\star}$ is weakly in $L^{1}(I, m)$, i.e. assume that for all $\varphi$ in $(\mathcal{S})$, the mapping $u \longmapsto \ll \Phi_{u}, \varphi \gg$, from $I$ to $\mathbb{R}$ belongs to $L^{1}(I, m)$. Then there exists an unique element in $(\mathcal{S})^{\star}$, denoted $\int_{I} \Phi_{u} m(d u)$, such that, for all $\varphi$ in $(\mathcal{S})$.

$$
\ll \int_{I} \Phi_{u} m(d u), \varphi \gg=\int_{I} \ll \Phi_{u}, \varphi \gg m(d u) .
$$

In this case, we say that $\Phi$ is $(\mathcal{S})^{\star}$ - integrable on $I$ (with respect to the measure $m$ ), in the Pettis sense. In the sequel, when we do not specify a name for the integral (resp. for the measure $m$ ) of an $(\mathcal{S})^{\star}$ - integrable process $\Phi$ on $I$, we always refer to the integral in Pettis's sense (resp. to the Lebesgue measure). 


\section{7. $\mathcal{S}$-transform and Wick product}

Lemma 1 ([16]). For any $(p, q) \in \mathbb{N}^{2}$ and $(X, Y) \in(\mathcal{S})_{-p} \times(\mathcal{S})_{-q}$,

$$
|\mathcal{S}(X \diamond Y)(\eta)| \leq\|X\|_{-p}\|Y\|_{-q} e^{|\eta|_{\max (p, q)}^{2}} .
$$

\section{Some properties of $\mathcal{S}$-transforms :}

(1) If $\Phi$ is deterministic function, then $\Phi \diamond \Psi=\Phi \Psi$, for all $\Psi \in(\mathcal{S})^{\star}$. Furthermore, let $\left(X_{t}\right)_{t \in \mathbb{R}}$ be a Gaussian process. If $U$ and $V$ two elements of $\mathcal{H}$, then

$$
U \diamond V=U V-\mathbb{E}[U V]
$$

(2) Let $\Phi=\sum_{k} a_{k}<\cdot, e_{k}>$ and $\Psi=\sum_{n} I_{n}\left(f_{n}\right)$ be in $(\mathcal{S})^{\star}$. So, their $\mathcal{S}$-transforms are given respectively as follows

$$
\mathcal{S}(\Phi)(\eta)=\sum_{k} a_{k}<\eta, e_{k}>_{L^{2}(\mathbb{R})}
$$

and

$$
\mathcal{S}(\Psi)(\eta)=\sum_{k}<f_{n}, \eta^{\otimes n}>
$$

for every $\eta \in \mathcal{L}(\mathbb{R})$.

(3) For every $(f, \eta, \xi)$ in $L^{2}(\mathbb{R}) \times \mathcal{L}(\mathbb{R}) \times \mathbb{R}$, we have

$$
(S)\left(e^{i \xi<\cdot, f>}\right)(\eta)=e^{\frac{1}{2}\left(|\eta|_{0}^{2}+2 i \xi<f, \eta>-\xi^{2}|f|_{0}^{2}\right)} .
$$

Also, the $\mathcal{S}$-transform verifies the following properties: see [Lebovits] [16].

\section{Lemma 2.}

(1) The map $\mathcal{S}: \Phi \longmapsto \mathcal{S}(\Phi)$, from $(\mathcal{S})^{\star}$ into $\mathcal{F}(\mathcal{L}(\mathbb{R}) \times \mathbb{R})$ is injective.

(2) Let $\Phi: I \longrightarrow(\mathcal{S})^{\star}$ be a $(\mathcal{S})^{\star}$ process. If $\Phi$ is $(\mathcal{S})^{\star}$-integrable on $I$ w.r.t $m$, then one has

$$
\forall \eta \in \mathcal{L}(\mathbb{R}), \mathcal{S}\left(\int_{I} \Phi(u) m(d u)\right)(\eta)=\int_{I} \mathcal{S}(\Phi(u))(\eta) m(d u)
$$

(3) Let $\Phi: I \longrightarrow(\mathcal{S})^{\star}$ be a $(\mathcal{S})^{\star}$ process. If $\Phi$ is $(\mathcal{S})^{\star}$-differentiable at $t \in I$, then, for every $\eta$ in $\mathcal{L}(\mathbb{R})$ the map $u \longmapsto[\mathcal{S} \Phi(u)](\eta)$ is differentiable at $t$ 
and verifies

$$
\mathcal{S}\left[\frac{d \Phi}{d t}(t)\right](\eta)=\frac{d}{d t}[\mathcal{S} \Phi(t)](\eta) .
$$

Theorem $1(15])$. Let $\Phi: I \longrightarrow(\mathcal{S})^{\star}$ be a stochastic distribution such that for all $\eta$ in $\mathcal{L}(\mathbb{R})$, the real-valued map $t \longmapsto \mathcal{S}[\Phi(t)](\eta)$ is measurable and such that there exist $p \in \mathbb{N}, a \in \mathbb{R}$ and a function $\mathcal{L}$ in $L^{1}(I, m)$ such that $|\mathcal{S}(\Phi(t))(\eta)| \leq \mathcal{L}(t) e^{a|\eta|_{p}^{2}}$, for all $\eta$ of $\mathcal{L}(R)$ and for almost every $t$ of $I$. Then $\Phi$ is $(\mathcal{S})^{\star}$-integrable on $I$, w.r.t to $m$.

Theorem 2 ([3] $)$. For any differentiable map $F: I \longrightarrow \mathcal{L}^{\prime}(\mathbb{R})$, the element $<\cdot F(t)>$ is a differentiable stochastic distribution process which satisfies the equality:

$$
\frac{d}{d t}<\cdot, F(t)>=<\cdot, \frac{d F}{d t}(t)>
$$

\subsection{Operators $\left(M_{H}\right)_{H \in(0,1)}$}

We now define our fundamental $L^{2}(\mathbb{R})$-operator $M_{H}$ for $0<H<1$, in the Fourier domain by:

$$
\widehat{M_{H}(u)}(y)=\frac{\sqrt{2 \pi}}{c_{H}}|y|^{1 / 2-H} \widehat{u}(y), \forall y \in \mathbb{R}^{\star},
$$

where $c_{H}$ is defined by

$$
c_{H}=\left(\frac{2 \pi}{\Gamma(2 H+1) \sin (\pi H)}\right)^{\frac{1}{2}} .
$$

We define the homogeneous Sobolev space of order $\frac{1}{2}-H$, noted $L_{H}^{2}(\mathbb{R})$ as:

$$
L_{H}^{2}(\mathbb{R}):=\left\{u \in \mathcal{S}^{\prime}(\mathbb{R}): \widehat{u}=T_{\varphi} ; \varphi \in L_{L o c}^{1}(\mathbb{R}) \text { and }\|u\|_{H}<+\infty\right\}
$$

where the norm derives from the inner product which is defined on $L_{H}^{2}(\mathbb{R})$, (for more details see [16]).

\section{A New Stochastic Integration}

In this part, we define our idea for stochastic integration ; we will integrate with respect to fractional Brownian motion a no adapted process, 
this later is a product of two processes, one is adapted, and the second is instantly independent.

Our work is based on a result of Ayed and Kuo [5], but in our case, we integrate with respect to fractional Brownian motion where the Hurst parameter $H$ is upper then $\frac{1}{2}$, and we append this work by a new integration of J.Lebovits [7].

Definition 3. A stochastic process $\phi(t)$ is called instantly independent process of $\left\{\mathcal{F}_{t}\right\}$ if $\phi(t)$ and $\mathcal{F}_{t}$ are independent for each $t \in[a, b]$.

Suppose $f(t)$ is an $\left\{\mathcal{F}_{t}\right\}$-adapted continuous stochastic process which is also $L^{2}$ - integrable. We present the following definition

Definition 4. For an adapted stochastic process $f(t)$ and an instantly independent stochastic process $\phi(t)$, we define the stochastic integral of $f(t) \phi(t)$ with respect to fractional Brownian motion $B_{t}^{H}$ as follows :

$$
\int_{a}^{b} f(t) \phi(t) d B_{t}^{H}=\lim _{\left\|\Delta_{n}\right\| \longrightarrow 0} \sum_{i=1}^{n} f\left(t_{i-1}\right) \phi\left(t_{i}\right)\left(B_{t_{i}}^{H}-B_{t_{i-1}}^{H}\right) .
$$

According to the stochastic integration w.r.t fractional Brownian motion introduced in [18], for $t \in[a, b]$ we have the following Wienner's Integration

$$
\int_{a}^{b} \phi(t) d B_{t}^{H}=\int_{a}^{b} \phi(t) K_{H}^{\star} d B_{t} .
$$

Hence, if $f(t) \phi(t) K_{H}^{\star} \in L^{2}[a, b]$, then this integral is well defined and we have

$$
\int_{a}^{b} f(t) \phi(t) d B_{t}^{H}=\int_{a}^{b} f(t) \phi(t) K_{H}^{\star} d B_{t} .
$$

Here, we integrate the product of two measurable processes with respect to standard Brownian motion $B_{t}$. Therefore, we find ourselves in the definition presented by Kuo et.al [9].

If we Take $\psi=f(t) K_{H}^{\star}$, we verify that this new process is also adapted.

By the above definition of $K_{H}^{\star}$, we can show that $\psi$ is adapted. Indeed, we have $f(t)$ is an $\left\{\mathcal{F}_{t}\right\}$-adapted stochastic process with almost all sample paths being in $L^{2}[a, b]$, moreover, $K_{H}^{\star}$ is an linear operator in $L^{2}[a, b]$. 
Therefore, the product of $f(t)$ and $K_{H}^{\star}$ are in $L^{2}[a, b]$, from it, we conclude that $\psi$ is an $\left\{\mathcal{F}_{t}\right\}$-adapted process with almost all sample paths being in $L^{2}[a, b]$.

We have given some properties of fractional Brownian motion, our aim is to define stochastic integral of the form

$$
\int_{a}^{b} F(t, \omega) d B_{t}^{H}
$$

where $B^{H}$ is fbm(fractional Brownian motion) and $F$ is non-adapted stochastic process, which is written as a product of two processes, one is adapted process and the second is instantly independent.

It's necessary to give some notions before explaining our approach.

Since $B^{H}$ is a Gaussian process, it easier for us to integrate with respect to this process (For more details see [16]). There remains the problem that the integrator is a non-adapted process.

The next definition from [16] is needed for our approach

Definition 5. Define for every $t$ in $\mathbb{R}$

$$
W_{t}^{(G)}=<\cdot, G_{t}^{\prime}>
$$

where the equality holds in $\left(\mathcal{S}^{\star}\right)$. Then $\left(W_{t}^{(G)}\right)_{t \in \mathbb{R}}$ is a $\left(\mathcal{S}^{\star}\right)$-process and is the $\left(\mathcal{S}^{\star}\right)$-derivative of the process $\left(G_{t}\right)_{t \in \mathbb{R}}$. We will sometimes $\frac{d G_{t}}{d t}$ instead of $W_{t}^{(G)}$.

By proposition 2.2 in [16], $W_{t}^{(G)}$ is defined by

$$
W_{t}^{(G)}=\sum_{k=0}^{+\infty}\left(\frac{d}{d t}<G_{t}, e_{k}>\right)<\cdot, e_{k}>.
$$

$\left\|W_{t}^{(G)}\right\|_{-p}$ is continuous if and only if $\left|G_{t}^{\prime}\right|_{-p}$ is continuous.

Remark 1 ([7]). The process $\left\|W_{t}^{\left(B^{H}\right)}\right\|_{-p}$ is continuous on the compact set $[0, T]$ for every $p \geq 2$ and $H \in(0,1)$.

As the fbm $B^{H}$ is continuous non-derivable, we define its increments for a time interval $s$. They can be assimilated to a derivative of the process at a 
resolution $s$. They are called fractional Gaussian noises. Such an increment is defined by

$$
G_{s}(i)=B^{H}(i)-B^{H}(i-s)
$$

Now, we introduce the integral of a non-adapted process $F$ w.r.t to fbm . Since the map $s \longrightarrow G_{s}$ is (weakly) differentiable on $I$, we give a Wienner's integral w.r.t $G_{s}$ as

$$
\begin{aligned}
\int_{I} F(s) d G_{s} & =\int_{I} f(s) \phi(s) d G_{s} \\
& =\int_{I} f(s) \phi(s) d\left[B^{H}(i)-B^{H}(i-s)\right] \\
& =\int_{I} f(s) \phi(s) d\left[\frac{B^{H}(i)-B^{H}(i-s)}{d s}\right] d s ; \\
& =\int_{I} f(s) \phi(s) W^{B_{i}^{H}-B_{i-s}^{H}} d s .
\end{aligned}
$$

Before passing to give assumption about a process $f(t)$ and $\phi(t)$, we need the next definition from [15];

Definition 6 (Bochner's Integral). Let $I$ be a subset of $[0,1]$ endowed with the Lebesgue's measure. One says that $\Phi: I \longrightarrow(\mathcal{S})^{\star}$ is Bochner's integrable of index $p$ on $I$ if it satisfies the two following conditions:

1. $\Phi$ is weakly measurable on $I$, i.e. $t \longrightarrow \ll \Phi_{t}, \varphi \gg$ is measurable on I for every $\varphi$ in $(\mathcal{S})$.

2. There exists $p$ in $\mathbb{N}$ such that $\Phi_{t} \in\left(\mathcal{S}_{-p}\right)$ for almost every $t$ in $I$ and such that $t \longrightarrow\left\|\Phi_{t}\right\|_{-p}$ belongs to $L^{1}(I, d t)$.

The Bochner's integral of $\Phi$ on $I$ is denoted $\int_{I} \Phi(t) d t$.

According to [16], we have this definition

Definition 7 (Wick-Itô's integral w.r.t Gaussian process). Let $X$ be a process with state space $(\mathcal{S})^{\star}$ such that the process $t \longmapsto X_{t} \diamond W_{t}^{G}$ is $(\mathcal{S})^{\star}$-integrable on $\mathbb{R}$. The process $X$ is then said to be $d G$-integrable on $\mathbb{R}$ 
w.r.t the Gaussian process $G$. The Wick-Itô's integral of $X$ w.r.t $G$, on $\mathbb{R}$, is defined by setting:

$$
\int_{\mathbb{R}} X_{s} d^{\diamond} G_{s}:=\int_{\mathbb{R}} X_{s} \diamond W_{s}^{(G)} d s .
$$

For any Borel set $I$ of $\mathbb{R}$, we also define $\int_{I} X_{s} d^{\diamond} G_{s}:=\int_{\mathbb{R}} I_{I}(s) X_{s} d^{\diamond} G_{s}$.

Now, back to our approach ; Let $H \in(0,1),[a, b]$ is Borel's subset of $(0,1) \cdot B^{H}=\left(B_{t}^{H}\right)_{t \in[a, b]}$ is a fbm with Hurst parameter $H \in[0,1]$. Assuming that $f(t) \phi(t)$ is an $(\mathcal{S})^{\star}$-valued process, we have the following results

(i) There exists $p \in \mathbb{N}$ such that $f(t) \phi(t) \in(\mathcal{S})^{\star}$ for a.e $t \in[a, b]$.

(ii) the process $f(t) \phi(t) \diamond W_{t}^{H}$ is Bochner's integrable on $[a, b]$.

Example 1. Let $\Delta=\left\{0=t_{0}, t_{1}, t_{2}, \cdots, t_{n}=1\right\}$ be a partition of the interval $[0,1]$. On the subinterval $\left[t_{i-1}, t_{i}\right]$, we take the right endpoint $t_{i}$ as the evaluation point for the integrand $B(1)-B(t)$. We define this integral

$$
\begin{aligned}
\int_{0}^{1} & (B(1)-B(t)) d^{\diamond} B^{H}(t) \\
& =\lim _{\|\Delta\| \rightarrow 0} \sum_{i=1}^{n}\left(B(1)-B\left(t_{i}\right)\right)\left(W_{t_{i}}^{B^{H}}-W_{t_{i-1}}^{B^{H}}\right) ; \\
& =B(1)^{2}-\lim _{\|\Delta\| \longrightarrow 0} \sum_{i=1}^{n} B\left(t_{i}\right)\left(W_{t_{i}}^{B^{H}}-W_{t_{i-1}}^{B^{H}}\right) ; \\
& =B(1)^{2}-\lim _{\|\Delta\| \longrightarrow 0} \sum_{i=1}^{n}\left\{\left[B\left(t_{i}\right)-B\left(t_{i-1}\right)\right]+B\left(t_{i-1}\right)\right\}\left(W_{t_{i}}^{B^{H}}-W_{t_{i-1}}^{B^{H}}\right) ; \\
& =B(1)^{2}-1-\int_{0}^{1} B(t) d^{\diamond} B^{H}(t)
\end{aligned}
$$

where the last integral is the Wick-Itô's integral.

We conclude that $\int_{0}^{1} B(1) d^{\diamond} B^{H}(t)=B(1)^{2}-1$.

In this way, we define an adapted process $f$ and an instantly independent process $\phi$ on $[0, T]$, with respect to $B^{H}$. Since the map $s \longrightarrow B_{s}^{H}$ is weakly 
differentiable on $[0, T]$, we present the formal definition of the Wienner's integral w.r.t $B^{H}$ denoted $\int_{[0, T]} f(s) \phi(s) d^{\diamond} B_{s}^{H}$, by setting

$$
\int_{[0, T]} f(s) \phi(s) d^{\diamond} B_{s}^{H}=\int_{[0, T]} f(s) \phi(s) \frac{d B_{s}^{H}}{d s} d s=\int_{[0, T]} f(s) \phi(s) W_{s}^{B^{H}} d s,
$$

In general, the problem of non-adaptation remains, in this case, we passe to give this definition

Definition 8. For an adapted stochastic process $f(t)$ and an instantly independent stochastic process $\phi(t)$, we define stochastic integral of $f(t) \phi(t)$ as follow

$$
\int_{[0, T]} f(s) \phi(s) d^{\diamond} B_{s}^{H}=\lim _{\left\|\Delta_{n}\right\| \longrightarrow 0} \sum_{i=1}^{n} f\left(t_{i-1}\right) \phi\left(t_{i}\right)\left(w_{t_{i}}^{B^{H}}-w_{t_{i-1}}^{B^{H}}\right),
$$

provided that the limit in probability exists.

\subsection{Some examples of our approach}

Example 2. For $t \in[0,1]$, we calculate the following stochastic integral using our approach ;

$$
\int_{0}^{t} B(1) B(s) d^{\diamond} B_{s}^{H}
$$

The random variable $B^{1}$ is independent of $B_{s}^{H}$ because $s$ is less than $t$, but the randon variable $B_{s}$ is adapted to the filtration generated by $B_{s}^{H}$ and so the randon variable $B(1) B(s)$ is not adapted to the filtration generated by $B_{s}^{H}$ and which is written as a product of two variables, one is instantly independent of $\left\{B_{s}^{H}, s \geq 0\right\}$ and the other is adapted to the filtration generated by $\left\{B_{s}^{H}, s \geq 0\right\}$.

By (13), this formula is given as

$$
\int_{0}^{t} B(1) B(s) d^{\diamond} B_{s}^{H}= \begin{cases}\frac{1}{2} B(1)\left(B(t)^{2}-t\right)-\int_{0}^{t} W_{s}^{B^{H}} d s, & 0<t \leq 1 \\ \frac{1}{2} B(1)\left(B(t)^{2}-t\right)-\int_{0}^{1} W_{s}^{B^{H}} d s, & t>1 .\end{cases}
$$


Example 3. Let $f(t)$ and $g(t)$ be two deterministic functions in $L^{2}([0,1])$. Then

$$
\int_{0}^{1} g(t)\left(\int_{0}^{1} f(s) d B(s)\right) d^{\diamond} B^{H}(t)=\int_{[0,1]^{2}} g(t) f(s) d B(s) \otimes d^{\diamond} B^{H}(t)
$$

Note that the Wiener integral of $f(s)$ in the left-hand side has the decomposition

$$
\int_{0}^{1} f(s) d B(s)=\int_{0}^{t} f(s) d B(s)+\int_{t}^{1} f(s) d B(s)
$$

while noticing that the first integral is in the itô part and the second integral is in the counterpart.

Let $W_{i}=W_{t_{i}}^{B^{H}}-W_{t_{i-1}}^{B^{H}}$. By Definition 8, we have

$$
\begin{array}{rl}
\int_{0}^{1} & g(t)\left(\int_{0}^{1} f(s) d B(s)\right) d^{\diamond} B^{H}(t) \\
= & \lim _{\|\Delta\| \rightarrow 0} \sum_{i=1}^{n} g\left(t_{i-1}\right)\left[\int_{0}^{t_{i-1}} f(s) d B(s)+\int_{t_{i}}^{1} f(s) d B(s)\right] \Delta W_{i} ; \\
= & \lim _{\|\Delta\| \longrightarrow 0} \sum_{i=1}^{n} g\left(t_{i-1}\right)\left[\int_{0}^{1} f(s) d B(s)-\int_{t_{i-1}}^{t_{i}} f(s) d B(s)\right] \Delta W_{i} ; \\
= & \int_{0}^{1} f(s) d B(s)-\lim _{\|\Delta\|} \sum_{i=1}^{n} g\left(t_{i-1}\right) f\left(t_{i-1}\right)\left[B\left(t_{i}\right)-B\left(t_{i-1}\right)\right] \Delta W_{i} ; \\
= & \int_{0}^{1} f(s) d B(s) \int_{0}^{1} g(t) d B^{H}(t) \\
& -\lim _{\|\Delta\| \longrightarrow 0} \sum_{i=1}^{n} g\left(t_{i-1}\right) f\left(t_{i-1}\right)\left[B\left(t_{i}\right)-B\left(t_{i-1}\right)\right] \Delta W_{i} ; \\
= & \int_{0}^{1} f(s) d B(s) \int_{0}^{1} g(t) d B^{H}(t)-\int_{0}^{1} g(t) f(t) W^{B^{H}}(t) d t
\end{array}
$$

\section{Conclusion}

The itô's integral is perfect for making sense and studying the differential systems driven by a semimartingale, so unfortunately we cannot use it in the case of the fractional Brownian motion. If $H>\frac{1}{2}$, the fractional 
has sufficiently regular trajectories to be able to use Young's integral, which is defined a bit like the Riemann integral, namely a passage to the limit after discretization. But the problem is going to complicate when the integrands are not adapted processes, in this case we take the wick-Itô's integral. In this paper, we introduced a new approach of stochastic integration for non-adapted processes with respect to fractional Brownian motion $\left(B_{s}^{H}\right)_{s \geq 0}$ (which is not a semimartingale for $H \neq \frac{1}{2}$ ). These processes are written as a product of two processes, one is instantly independent of $B_{s}^{H}$ and the other is adapted to the filtration generated by $\left\{B_{s}^{H}, s \geq 0\right\}$.

\section{References}

1. E. Alòs, O. Mazet and D. Nualart, Stochastic calculus with respect to fractional Brownian motion with Hurst parameter lesser than $\frac{1}{2}$, Stoch. Proc. Appl., 86(2000), 121-139.

2. Ben McGonegal, Fractional Brownian Motion and the Fractional Stochastic Calculus, arXiv:1402.2752.2014.

3. C. Bender, An Itô formula for generalized functionals of a fractional Brownian motion with arbitrary Hurst parameter, Stochastic Processes and Their Applications, 104(2003), 81-106.

4. E. Alòs, J. A. León and D. Nualart, Stratonovich stochastic calculus with respect to fractional Brownian motion with Hurst parameter less than $\frac{1}{2}$, Taiwanesse Journal of Mathematics, 2000.

5. W. Ayed and H.-H. Kuo, An extension of the Itô integral, Theory of Stochastic Processes, 16(32), no. 1, 2010, pp. 3, 17-28.

6. F. Baudoin and David Nualart, Equivalence of Volterra processes, Stochastic Process. Appl., 107(2003), No. 2, 327-350.

7. J. Lebovits and J. Lvy Vhel, White noise-based stochastic calculus with respect to multifractional Brownian motion, Stoch. Proc. Appl., 86 (2014), No.1, 87-124.

8. P. Carmona and L. Coutin, Stochastic integration with respect to fractional Brownian motion, Preprint 1998.

9. Chii-Ruey Hwang. Hui-Hsiung Kuo. Kimiaki Sait and Jiayu Zhai, A general Itô formula for adapted and instantly independent stochastic processes, Communications on Stochastic Analysis, 10(2016), No. 3, Article 5,2016.

10. P. Cheridito, Mixed fractional Brownian motion, Bernoulli 7(2001), 913-934.

11. Z. Ciesielski, G. Kerkyacharian and B.Roynette, Quelques espaces fonctionnels associés a des processus guassiens. (Some function spaces associated with gaussian processes)., Stud. Math., 107(1993), 171-204. 
12. F. Comte and E. Renault, long memory continuous time models, Journal of Econometrics, 73(1996), 101-150.

13. L. Decreusefond and A. S. Üstünel, Stochastic analysis of the fractional Brownian motion, Potential Analysis, 10(1998), 177-214.

14. A. N. Kolmogorov, Wienersche Spiralen und einige andere interessante Kurven im Hilbertschen Raum, C.R. (Doklady) Acad. URSS (N.S), 26(1940), 115-118.

15. H. Kuo, White Noise Distribution Theory, CRC-Press, 1996.

16. J. Lebovits, Stochastic Calculus with respect to Gaussian processes, May 22, 2015.arXiv.1408.1020v2.

17. Benoit B. Mandelbrot and John W. Van Ness, Fractional Brownian motions, fractional noises and applications, SIAM Rev., 10(1968), 422-437.

18. Joachim Y. Nahmani, Introduction to Stochastic Integration with respect to Fractional Brownian Motion, June 2009.

19. S. J. Lin, Stochastic analysis of fractional Brownian motions, Stoch. Stoch. Rep., 55(1995), 121-140.

20. T. E. Duncan, Y. Hu and B. Pasik-Duncan, Stochastic calculus for fractional Brownian motion I, Theory SIAM J. Control Optim., 38(2000), 582-612.

21. W. Dai and C. C. Heyde, Itô Formula with respect to fractional Brownian motion and its application, Journal of Applied Mathematics and Stochastic Analysis, Anal. 9(1996), 439-448. 